\section{REFERENCES}

1. Akelma AZ, Mete E, Cizmeci MN, Kanburoglu MK, Malli DD, Bozkaya D. The role of mean platelet volume as an inflammatory marker in children with chronic spontaneous urticaria. Allergol Immunopathol (Madr) 2015;43:10-13.

2. Chandrashekar L, Rajappa M, Sundar I, Munisamy M, Ananthanarayanan $\mathrm{PH}$, Thappa DM, et al. Platelet activation in chronic urticaria and its correlation with disease severity. Platelets 2014;25:162-165.

3. Confino-Cohen R, Chodick G, Shalev V, Leshno M, Kimhi $\mathrm{O}$, Goldberg A. Chronic urticaria and autoimmunity: associations found in a large population study. J Allergy Clin Immunol 2012;129:1307-1313.

4. Isiksacan N, Koser M, Cemsitoglu F, Kucuksezer UC, Gurdol F. Platelet and other hemostatic characteristics in patients with chronic urticaria. Angiology 2015;66:387-391.

5. Kim DS, Lee J, Kim SH, Kim SM, Lee MG. Mean platelet volume is elevated in patients with psoriasis vulgaris. Yonsei Med J 2015;56:712-718.

6. Topal E, Celiksoy MH, Catal F, Karakoç HT, Karadağ A, Sancak $\mathrm{R}$. The platelet parameters as inflammatory markers in preschool children with atopic eczema. Clin Lab 2015; 61:493-496.

7. Bai M, Xing L, Feng J, Cui C, Huang L, Liang G. Mean platelet volume could reflect disease activity of adult patients with systemic lupus erythematosus. Clin Lab 2016;62: 1317-1322.

8. Gasparyan AY, Ayvazyan L, Mikhailidis DP, Kitas GD. Mean platelet volume: a link between thrombosis and inflammation? Curr Pharm Des 2011;17:47-58.

\title{
Comparative Study of Clinical Characteristics according to Therapeutic Efficacy and Drug Survival of Cyclosporine or Methotrexate in Psoriasis Vulgaris
}

\author{
Hyun Soo Lee, Soo Hyun Kwon, Eun-So Lee \\ Department of Dermatology, Ajou University School of Medicine, Suwon, Korea
}

Dear Editor:

Psoriasis vulgaris is a chronic, multifactorial inflammatory skin disease. Cyclosporine (CsA) and methotrexate (MTX) are conventional but still highly effective therapeutic options for patients with psoriasis vulgaris, as not all patients are appropriate for biological therapy.

Since the introduction of the tumor necrosis factor (TNF) inhibitors and other biological agents for the treatment of psoriasis, CsA and MTX have become less frequently discussed $^{1}$. In particular, no studies have focused on the clinical and laboratory factors to predict therapeutic response to CsA or MTX. This study aimed to compare clinical and laboratory characteristics according to therapeutic response to either CsA or MTX. Furthermore, we aimed to demonstrate the predictive factors of therapeutic response to either CsA or MTX.

\section{Received July 23, 2018, Revised October 10, 2018, Accepted for publication October 26, 2018}

Corresponding author: Eun-So Lee, Department of Dermatology, Ajou University School of Medicine, 164 WorldCup-ro, Yeongtong-gu, Suwon 16499, Korea. Tel: 82-31-219-5190, Fax: 82-31-219-5189, E-mail: esl@ajou.ac.kr ORCID: https://orcid.org/0000-0003-0232-7704

This is an Open Access article distributed under the terms of the Creative Commons Attribution Non-Commercial License (http://creativecommons.org/ licenses/by-nc/4.0) which permits unrestricted non-commercial use, distribution, and reproduction in any medium, provided the original work is properly cited.

Copyright (C) The Korean Dermatological Association and The Korean Society for Investigative Dermatology 
The study was approved by the institutional review board of Ajou University Hospital (IRB no. AJIRB-BMR-MDB-15341).

Patients with psoriasis vulgaris who had been treated with either CsA or MTX at the dermatology department of Ajou University Hospital between January 2012 and December 2016 were enrolled. The patients were divided into four subgroups, namely CsA responders, MTX responders, CsA non-responders, and MTX non-responders. CsA or MTX responders were defined as patients treated with a single systemic agent, either CsA or MTX, during the study period. CsA or MTX non-responders were defined as patients whose single-agent treatment was switched to another agent such as acitretin, which is rarely prescribed for treating psoriasis in our dermatology department. Their electronic medical records were retrospectively reviewed by single dermatologist, including age, sex, disease duration, duration of each medication course, body mass index (BMI), initial erythrocyte sedimentation rate (ESR), and initial C-reactive protein (CRP) level. These clinical and laboratory variables were statistically compared among the four groups.

Additional study was conducted to determine whether drug survival of either CsA or MTX was associated with the clinical and laboratory factors. Drug survival is defined as the time from initiation to discontinuation of treatment. Drug survival of CsA was investigated in the combined CsA responder and non-responder group. In addition, MTX responders and non-responders were combined to investigate the drug survival of MTX.

Statistical analysis was performed using $\mathrm{R}$ software packages ( $R$ version 3.4.1). Generalized linear model and Tukey contrasts were used to compare the variables among the four groups. The Bonferroni correction method was used to avoid a type I error and adjust the $p$-value. Significance was assumed at $p \leq 0.05$. The Kaplan-Meier curve, logrank tests, and Cox and logistic regression analysis were used for drug survival analysis. To stratify the Kaplan-
Meier drug survival curve, each continuous variable was converted into a binary variable. The cutoff values were as follows: disease duration, 36 months; BMI, $23 \mathrm{~kg} / \mathrm{m}^{2}$; ESR, $20 \mathrm{~mm} / \mathrm{h}$; and CRP level, $0.05 \mathrm{mg} / \mathrm{dl}$.

For this study, 488 patients with psoriasis vulgaris were enrolled. The mean patient age was 42.6 years, and the total sex ratio (male-to-female) was 1.8:1. The patients consisted of 199 CsA responders, 199 MTX responders, 53 CsA non-responders, and 37 MTX non-responders (Table 1). The mean ages in each group were 43.6, 42.5, 38.6, and 44.0 years, respectively. The CsA responders had the shortest disease duration among all the groups (45.1 vs. 80.8, 65.6, and 115.1 months, respectively). The disease duration of the CsA responders was significantly shorter than that of the MTX responders and MTX non-responders (Fig. 1A). The non-responders to either CsA or MTX had a longer disease duration than the responders, although the difference was not statistically significant. BMI did not show significant differences among the groups. The CsA responders showed the lowest initial serum ESR level (10.1 vs. 11.3, 13.2, and $17.7 \mathrm{~mm} / \mathrm{h}$, respectively). The difference was statistically significant between the CsA responders and MTX non-responders (Fig. 1B). The non-responders to either CsA or MTX tended to have a higher initial serum ESR level than the responders. The initial serum CRP level did not differ significantly among the groups.

The drug survival of CsA was significantly longer in the patients with disease durations of $>36$ months $(p \leq 0.05)$. Otherwise, no significant differences in clinical factors were observed. Moreover, the results demonstrated that all the clinical factors had no influence on the drug survival of MTX.

This was a pilot study to investigate the predictive factors of clinical responses to either CsA or MTX. The results revealed that the CsA responders had the shortest disease duration among the four groups. CsA was suggested to be more effective in the patients with a shorter history of psoriasis.

Table 1. Characteristics of the patients

\begin{tabular}{lcccc}
\hline \multicolumn{1}{c}{ Characteristic } & CsA responders & MTX responders & CsA non-responders & MTX non-responders \\
\hline Age $(\mathrm{yr})$ & $43.6 \pm 1.2$ & $42.5 \pm 1.1$ & $38.6 \pm 2.0$ & $44.0 \pm 2.8$ \\
Sex ratio (male-to-female) & $1.8: 1$ & $1.9: 1$ & $1.7: 1$ & $1.5: 1$ \\
Treatment duration (mo) & $6.4 \pm 0.7$ & $7.0 \pm 0.7$ & $9.9 \pm 1.5^{*} / 9.1 \pm 1.3^{\dagger}$ & $26.0 \pm 5.5^{\dagger} / 7.5 \pm 1.8^{*}$ \\
Disease duration (mo) & $45.1 \pm 5.8$ & $80.8 \pm 7.4$ & $65.6 \pm 13.7$ & $115.1 \pm 22.8$ \\
BMI $\left(\mathrm{kg} / \mathrm{m}^{2}\right)$ & $23.9 \pm 0.4$ & $24.7 \pm 0.4$ & $23.0 \pm 0.7$ & $24.3 \pm 0.7$ \\
Initial ESR (mm/h) & $10.1 \pm 1.1$ & $11.3 \pm 0.9$ & $13.2 \pm 3.1$ & $17.7 \pm 3.0$ \\
Initial CRP (mg/dl) & $0.3 \pm 0.1$ & $0.3 \pm 0.1$ & $0.7 \pm 0.6$ & $0.8 \pm 0.5$ \\
\hline
\end{tabular}

Values are presented as mean \pm standard deviation or number only. CsA: cyclosporine, MTX: methotrexate, BMI: body mass index, ESR: erythrocyte sedimentation rate, CRP: C-reactive protein. ${ }^{*} \mathrm{Cs} A,{ }^{\dagger} \mathrm{MTX}$. 
A

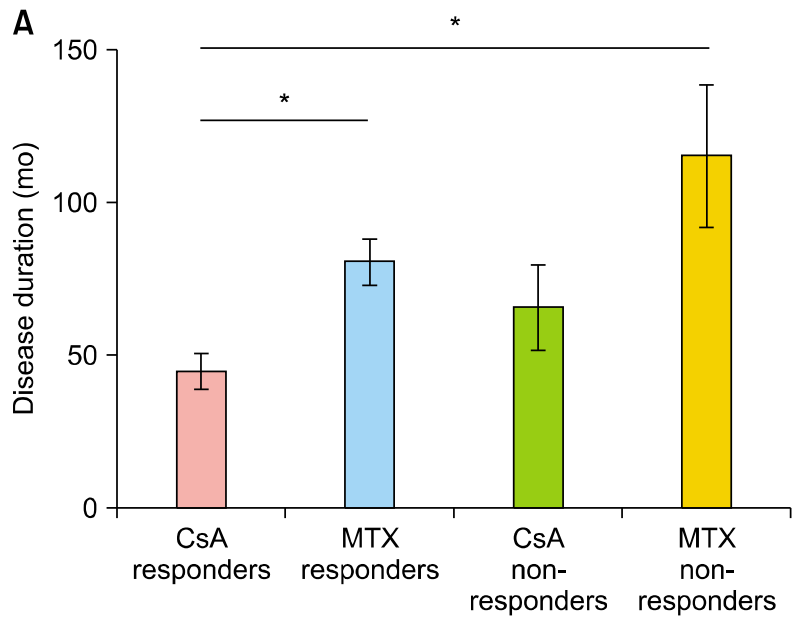

B

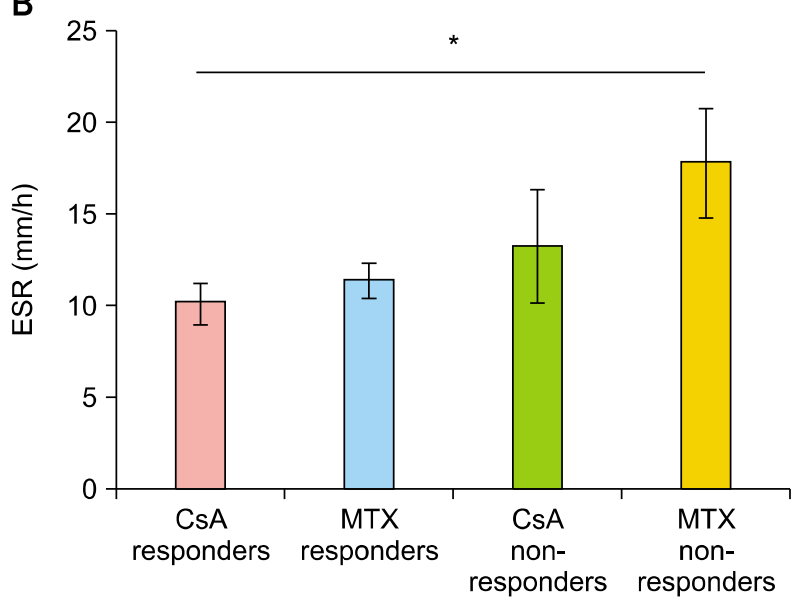

Fig. 1. (A) Cyclosporine $(\mathrm{Cs} A)$ responders had the shortest disease duration among the methotrexate (MTX) responders, CsA non-responders, and MTX non-responders (45.1 vs. 80.8, 65.6, and 115.1 months, respectively). The disease duration of the CsA responders was significantly shorter than that of the MTX responders and non-responders (marked with asterisk). (B) The CsA responders showed the lowest initial serum erythrocyte sedimentation rate (ESR) level (10.1 vs. 11.3, 13.2, and $17.7 \mathrm{~mm} / \mathrm{h}$, respectively). The difference was statistically significant between the CsA responders and MTX non-responders (marked with asterisk).

Among the patients who were receiving CsA, those with longer disease duration ( $>36$ months) maintained CsA for a longer time. Generally, longer treatment duration means good response to the treatment. However we assumed that the patients with longer disease duration tended to be less responsive to $\mathrm{CsA}$, so the treatment duration became longer.

Various serological markers of inflammation have been used to assess disease activity and response to treatment. ESR is known to be good for long-term monitoring, whereas CRP levels reflect day-to-day variations of disease activity $^{2}$. In our study, the CsA responders showed the lowest initial serum ESR level, suggesting the lowest inflammatory level.

The baseline patient characteristics associated with the effectiveness of biologics that were identified in previous studies were baseline BMI, baseline psoriasis activity and severity index (PASI), disease duration, and biological naivety $^{3-7}$. However, baseline BMI did not show significance in our study.

One of the limitations of our study is its retrospective design based on reviewing medical charts. Second, PASI data were not included. Therefore, the exact severity of psoriasis could not be defined and therapeutic responsiveness was not objectified. Third, in defining non-responders, the fact that non-responders might have changed their first drug due to side effects was ignored. However, it is still meaningful to include a large number of subjects.

In conclusion, shorter disease duration and low initial serum ESR level might predict good responsiveness to CsA in psoriasis. This pilot study is the first study to evaluate the clinical and laboratory factors that might influence the therapeutic responsiveness and drug survival of CsA and MTX.

\section{ACKNOWLEDGMENT}

This research was supported by a grant of the Korea Health Technology R\&D Project through the Korea Health Industry Development Institute (KHIDI), funded by the Ministry of Health \& Welfare, Republic of Korea (grant number: HI16C0992).

\section{CONFLICTS OF INTEREST}

The authors have nothing to disclose.

\section{ORCID}

Hyun Soo Lee, https://orcid.org/0000-0001-7715-7688 Soo Hyun Kwon, https://orcid.org/0000-0002-2336-9770 Eun-So Lee, https://orcid.org/0000-0003-0232-7704

\section{REFERENCES}

1. Strober BE. Methotrexate and cyclosporine in psoriasis revisited. Semin Cutan Med Surg 2014;33(2 Suppl 2):S27S30.

2. Kanelleas A, Liapi C, Katoulis A, Stavropoulos P, Avgerinou $G$, Georgala $S$, et al. The role of inflammatory markers in assessing disease severity and response to treatment in patients with psoriasis treated with etanercept. Clin Exp Der- 
Brief Report

matol 2011;36:845-850.

3. Zweegers J, Roosenboom B, van de Kerkhof PC, van den Reek JM, Otero ME, Atalay S, et al. Frequency and predictors of a high clinical response in patients with psoriasis on biological therapy in daily practice: results from the prospective, multicenter BioCAPTURE cohort. Br J Dermatol 2017; 176:786-793.

4. Strober BE, Bissonnette R, Fiorentino D, Kimball AB, Naldi $L$, Shear $N H$, et al. Comparative effectiveness of biologic agents for the treatment of psoriasis in a real-world setting: results from a large, prospective, observational study (Psoriasis Longitudinal Assessment and Registry [PSOLAR]). J Am Acad Dermatol 2016;74:851-861.e4.

5. Menting SP, Sitaram AS, Bonnerjee-van der Stok HM, de
Rie MA, Hooft L, Spuls PI. Drug survival is not significantly different between biologics in patients with psoriasis vulgaris: a single-centre database analysis. Br J Dermatol 2014;171: 875-883.

6. van Lümig PP, van de Kerkhof PC, Boezeman JB, Driessen $\mathrm{RJ}$, de Jong EM. Adalimumab therapy for psoriasis in real-world practice: efficacy, safety and results in biologicnaïve vs. non-naïve patients. J Eur Acad Dermatol Venereol 2013;27:593-600.

7. Shear NH, Hartmann M, Toledo-Bahena $M$, Katsambas A, Connors L, Chang Q, et al.; REALITY investigators. Longterm efficacy and safety of infliximab maintenance therapy in patients with plaque-type psoriasis in real-world practice. Br J Dermatol 2014;171:631-641.

\title{
Senile Purpura: Clinical Features and Related Factors
}

\author{
Soo Ick Cho, Ji Won Kim, Gyeongyeon Yeo ${ }^{1}$, Dongmuk Choi ${ }^{1}$, Junggyo Seo ${ }^{1}$, Hyun-Sun Yoon ${ }^{2}$, \\ Jin Ho Chung
}

Department of Dermatology, Seoul National University College of Medicine, ${ }^{1}$ Seoul National University College of Medicine, ${ }^{2}$ Department of Dermatology, SMG-SNU Boramae Medical Center, Seoul, Korea

\section{Dear Editor:}

Senile or actinic purpura commonly presents as purpuric macules and patches on the sun-exposed skin in elderly individuals. The prevalence of senile purpura is approximately $10 \%$ in elderly individuals ${ }^{1}$. Senile purpura is considered a consequence of skin aging primarily attributable to photodamage and is often called dermatoporosis ${ }^{2}$. Although it is a common and clinically important condition, only a few studies have described in detail the clinical features or factors related to senile purpura ${ }^{3}$.

We performed a cross-sectional study using questionnaires to assess the clinical features and other factors related to senile purpura at Seoul National University Hospital (SNUH) and 20 local senior welfare centers between October and December 2017. The study protocol was approved by the Institutional Review Board of SNUH (IRB no. 1708-137-879), and written informed consent was obtained from all participants.

Patients enrolled in the study were asked whether they have/had senile purpura. Using a digital thickness micrometer (Mitutoyo Corporation, Kanagawa, Japan), double fold of skin thickness was measured at the level of the extensor area of the right lower arm $15 \mathrm{~cm}$ away from the elbow.

\footnotetext{
Received October 18, 2018, Accepted for publication November 1, 2018
}

Corresponding author: Jin Ho Chung, Department of Dermatology, Seoul National University Hospital, 101 Daehak-ro, Jongno-gu, Seoul 03080, Korea. Tel: 82-2-2072-2410, Fax: 82-2-742-7344, E-mail: jhchung@snu.ac.kr ORCID: https://orcid.org/0000-0002-0582-6392

This is an Open Access article distributed under the terms of the Creative Commons Attribution Non-Commercial License (http://creativecommons.org/ licenses/by-nc/4.0) which permits unrestricted non-commercial use, distribution, and reproduction in any medium, provided the original work is properly cited.

Copyright $($ The Korean Dermatological Association and The Korean Society for Investigative Dermatology 